\title{
WEAK TOPOLOGIES ON THE BOUNDED HOLOMORPHIC FUNCTIONS
}

\author{
BY L. A. RUBEL ${ }^{1}$ AND A. L. SHIELDS ${ }^{2}$ \\ Communicated by W. Rudin, August 17, 1964
}

Let $G$ be a region in the complex plane such that there is a nonconstant bounded holomorphic function on $G$, and denote the algebra of all such functions by $B_{H}(G)$. Let $H_{\infty}(G)$ denote the Banach algebra that arises when $B_{H}(G)$ is endowed with the supremum norm. In the case where $G$ is the unit disc $D, H_{\infty}(G)$ has been extensively studied, mostly by a real-variables analysis of the radial boundary values of bounded holomorphic functions.

We consider here another natural topology on $B_{H}(G)$, namely, the strict topology $\beta$, introduced by Buck in [2], where he made a preliminary study of the case $G=D$. In the work that we outline in this announcement, a number of intrinsic complex-variables methods are introduced, and used in conjunction with the theory of topological linear spaces to investigate the structure of $\beta(G)$, and to shed some light also on the structure of $H_{\infty}(G)$. Some of the general results obtained here were obtained in a different form, in the special case $G=D$, by Brown, Shields, and Zeller [1].

One of our results is that $H_{\infty}(G)$ is always the dual of a certain Banach space of equivalence classes of measures. Letting $\alpha(G)$ denote $B_{H}(G)$ under the weak topology arising from this duality, we exhibit strong similarities between $\alpha(G)$ and $\beta(G)$ that can be exploited in studying the ideal structure of $\beta(G)$.

The main new tool is the balayage, or sweeping, of measures. In point of fact, several different methods of balayage are used. One is similar to the balayage of potential theory, another uses duality of certain topological vector spaces, and the third uses ideas related to the Cauchy integral formula.

We present here only our main results, without proof. The full details will be published elsewhere. The order in which the results are presented is a little artificial, since some of the structure theorems depend on the balayage theorems.

\section{Structure.}

Definition. $\beta(G)$ is $B_{H}(G)$ under the topology given by the seminorms

\footnotetext{
${ }^{1}$ The research of this author was partially supported by an Air Force grant.

2 The research of this author was partially supported by the National Science Foundation.
} 


$$
\|f\|_{k}=\sup \{|f(z) k(z)|: z \in G\},
$$

where $k$ runs over the class of all continuous complex-valued functions on the closure of $G$ that vanish on the boundary of $G$.

Definition. To say that a (complex) measure $\mu$ lives in $G$ means that $\mu$ has no mass in the complement of $G$.

Definition. $M(G)$ is the Banach space of all bounded Borel measures $\mu$ that live in $G$, with

$$
\|\mu\|=\int d|\mu|,
$$

where $|\mu|$ is the variation of $\mu$.

Definition. We write $\mu \sim \nu$ for $\mu, \nu \in M(G)$ to mean that $\int f d \mu=\int f d \nu$ for each $f \in B_{H}(G)$, we write $[\mu]$ for the equivalence class that $\mu$ belongs to, and we write $\int f d[\mu]$ for $\int f d \mu$ if $f \in B_{H}(G)$.

Definition. $M^{\prime}(G)$ is the Banach space of all equivalence classes $[\mu]$ of measures $\mu$ in $M(G)$, with the quotient norm $\|[\mu]\|=\inf \{\|\nu\|: \nu \sim \mu\}$.

Definition. $\alpha(G)$ is $B_{H}(G)$ under the weak topology arising from the duality $\left\langle B_{H}(G), M^{\prime}(G)\right\rangle$, where for $f \in B_{H}(G)$ and $[\mu] \in M^{\prime}(G)$,

$$
\langle f,[\mu]\rangle=\int f d \mu .
$$

Theorem. $\alpha(G)$ and $\beta(G)$ have the same dual space, namely $M^{\prime}(G)$, with the duality given by (1).

Corollary. $\alpha(G)$ and $\beta(G)$ have the same closed subspaces, and the same closed convex sets.

TheOREM. $\alpha(G)$ and $\beta(G)$ have the same bounded sets, namely, the uniformly bounded sets of holomorphic functions.

TheOREM. $\alpha(G)$ and $\beta(G)$ have the same compact sets. In both $\alpha(G)$ and $\beta(G)$, a set is compact if and only if it is closed and bounded.

TheOREM. $\alpha(G)$ and $\beta(G)$ have the same convergent sequences; $a$ sequence $\left\{f_{n}\right\}$ is convergent if and only if the $f_{n}$ are uniformly bounded, and $\left\{f_{n}\right\}$ converges at each point of $G$.

Theorem. A linear subspace of $\alpha(G)$ or $\beta(G)$ is closed if and only if it is sequentially closed.

TheOREM. $\alpha(G)$ and $\beta(G)$ are not the same topologies; in fact, $\beta(G)$ is properly stronger than $\alpha(G)$. Also, $\beta(G)$ is complete, but $\alpha(G)$ is not.

It is easy to see that $\beta(G)$ is a topological algebra. We do not know whether this is true of $\alpha(G)$. 


\section{Balayage.}

DEFINITION. Let $L_{1}(G)$ consist of all measures $\mu \in M(G)$ that are absolutely continuous with respect to planar Lebesgue measure, with $\|\mu\|=\int d|\mu|$ as before.

Definition. Let $L_{1}^{\prime}(G)$ be the quotient space $L_{1}^{\prime}(G)=L_{1}(G) / N$, where $N=\left\{\mu \in L_{1}(G): \mu \sim 0\right\}$.

Theorem. Given any measure $\mu \in M(G)$ and any $\epsilon>0$, there exists a measure $\nu \in L_{1}(G)$ such that $\nu \sim \mu$ and $\|\nu\|<(1+\epsilon)\|\mu\|$.

Corollary. $M^{\prime}(G)$ is isometrically isomorphic to $L_{1}^{\prime}(G)$.

Corollary. $M^{\prime}(G)$ is separable.

The above theorem is useful because although $M(G)$ is a somewhat pathological space, $L_{1}(G)$ is a familiar space with convenient properties; in particular, the dual of $L_{1}(G)$ is the familiar space $L_{\infty}(G)$.

Theorem. $H_{\infty}(G)$ is the dual of $M^{\prime}(G)$. More precisely, to each $f \in H_{\infty}(G)$, there corresponds the continuous linear functional $L_{f}$ in the dual of $M^{\prime}(G)$, given by

$$
L_{f}([\mu])=\int f d \mu
$$

and the mapping $f \rightarrow L_{f}$ is an isometric isomorphism of $H_{\infty}(G)$ onto the dual of $M^{\prime}(G)$.

Definition. $A$ subset $S$ of $G$ is called dominating provided that

$$
\sup \{|f(z)|: z \in S\}=\sup \{|f(z)|: z \in G\}
$$

for each $f \in B_{H}(G)$.

Definition. $A$ subset $S$ of $G$ is called universal provided that for each $\mu \in M(G)$, there is a $\nu \in M(G)$ with $\nu \sim \mu$, such that $\nu$ lives on $S$.

Theorem. $A$ subset $S$ of $G$ is dominating if and only if it is universal.

Definition. Given an open set $G^{\prime}$, and a closed subset $E$ of $G^{\prime}$, let $G=G^{\prime}-E$. We say that $E$ is a set of removable singularities for $B_{H}(G)$ provided that each $f \in B_{H}(G)$ has an extension $F \in B_{H}\left(G^{\prime}\right)$.

Definition. Given $G^{\prime}$ and $E$ as above, we say that a measure $\mu \in M(G)$ is holomorphically free of $E$ to mean that for some $\epsilon>0$, there exists a measure $\nu$ that lives in the set

$$
E_{\mathrm{e}}=\{z \in G: \text { distance }(z, E)>\epsilon\}
$$

and such that $\nu \sim \mu$.

Theorem. Given $G^{\prime}$ and $E$ as above, $E$ is a set of removable singulari- 
ties for bounded holomorphic functions if and only if each measure $\mu \in M(G)$ is holomorphically free of $E$.

THEOREM. Suppose $S$ is a subset of $G$ with no limit points in $G$. Then $S$ is universal if and only if there is a measure $\nu \in M(G)$ that lives in $S$, with $\nu \sim 0$ but $\nu \neq 0$.

The method of proof of this result gives an explicit balayage formula.

Ideals in $\beta(G)$.

THEOREM. If $G$ is the open unit disc $D$, then the ideal generated by $f$ is dense in $\beta(G)$ if and only if $f$ is an outer function, and the ideal generated by $f$ is closed if and only if $f$ is the product of an inner function and a unit.

The first part of this result answers a question raised by Buck [2]. We have been told that Paul Hessler has a proof of the second part of this result. It is clear that the units in $\beta(G)$ are just those $f \in \beta(G)$ which are bounded away from 0 . The above theorem suggests the following definitions.

DEFINITION. In $\beta(G)$, interior functions are those that generate closed ideals, and exterior functions are those that generate dense ideals.

Problem. For what regions $G$ does each $f \in \beta(G)$ have a unique factorization, modulo units, as the product of an interior function and an exterior function?

THEOREM. If $\partial G$ is the union of nondegenerate continua and isolated points, then the only continuous multiplicative linear functionals on $\beta(G)$ are the point evaluations at points of $G$ and at removable singularities of $G$.

Rudin [3] has given examples of regions $G$ such that $\beta(G)$ has other continuous multiplicative linear functionals than the ones described above. It seems likely that each closed ideal in $\beta(G)$ is principal, but we have not been able to prove this, except in the case $G=D$.

\section{REFERENCES}

1. L. Brown, A. Shields and K. Zeller, On absolutely convergent exponential sums, Trans. Amer. Math. Soc. 96 (1960), 162-183.

2. R. C. Buck, Algebraic properties of classes of analytic functions, Seminars on Analytic Functions, Vol. II, pp. 175-188, Institute for Advanced Study, Princeton, N. J., 1957. 324.

3. W. Rudin, Essential boundary points, Bull. Amer. Math. Soc. 70 (1964), 321-

UNIVERSITY OF ILLINOIS AND

UNIVERSITY OF MICHIGAN 